\title{
Arrayed Space Optical Communications: Localization of the Ground Station
}

\author{
Zexi Fang and Athanassios Manikas \\ Department of Electrical and Electronic Engineering \\ Imperial College London \\ \{zexi.fang12, a.manikas\}@imperial.ac.uk
}

\begin{abstract}
In this paper, a novel ground station localization algorithm is proposed for space optical communications using array processing and a set of celestial objects of known locations in the global coordinate system. First, the ground station estimates the directions of this set of celestial objects relative to its local coordinate system using the sunlight reflected by these celestial objects. Then, the ranges of the celestial objects and the location and orientation of the ground station are estimated by solving systems of nonlinear and linear equations. The performance of the proposed approach is assessed through computer simulation studies. It is shown to estimate the location and orientation of the ground station successfully with excellent accuracy.

Index Terms-Array processing, space optical communications, ground station, localization, orientation estimation.
\end{abstract}

$\begin{array}{ll}a, A & \text { Scalar } \\ \underline{a}, \underline{A} & \begin{array}{l}\text { Column vector } \\ \mathbb{A}\end{array} \\ (\cdot)^{T} & \text { Matrix } \\ (\cdot)^{H} & \text { Transpose } \\ (\cdot)^{\#} & \text { Hermitian } \\ \|\cdot\| & \text { Moore-Penrose pseudoinverse } \\ \otimes & \text { Euclidean norm } \\ \operatorname{diag}(\underline{A}) & \text { Kronecker product } \\ \exp (\underline{A}) & \text { Diagonal matrix formed from } \underline{A} \\ \operatorname{vec}(\underline{\mathbb{A}}) & \text { Column-wise vectorization of } \underline{A} \\ \mathcal{E}\{\cdot\} & \text { Expectation operator } \\ \underline{1}_{N} & \text { Column vector of } N \text { ones } \\ \underline{0}_{N} & \text { Column vector of } N \text { zeros } \\ \mathbb{I}_{N} & N \times N \text { identity matrix } \\ \mathcal{R} & \text { Set of real numbers } \\ \mathcal{C} & \text { Set of complex numbers }\end{array}$

\section{INTRODUCTION}

Recently, there has been an increasing interest in exploring outer space. As a consequence, high-speed data transmission is required between Earth and space for a large amount of data exchange. This demand necessitates a broad channel bandwidth, which cannot be sufficiently provided within the traditional radio frequency (RF) band. Therefore, the optical band has been exploited in space communications because of its unlimited and unregulated spectrum [1]-[4]. Besides its large bandwidth, the optical band also offers other benefits, such as the reduction in the mass and cost of spacecraft.
Along with these advantages, one of the major challenges posed in space optical communications is the establishment of a stable data link between the ground station on Earth and spacecraft in space. Since the beamwidth of optical sources (e.g., laser sources) is restricted by the diffraction from their aperture and is extremely small in general, a subtle uncertainty in the beam direction can lead to significant power loss and performance deterioration. Thus, an accurate beam direction (e.g., with the error smaller than $1 \mu \mathrm{rad}$ ) is a prerequisite for successful data transmission using optical signals [4]. In order to achieve such high precision in beam direction, the locations of the ground station and spacecraft need to be accurately estimated prior to the establishment of a data link [4].

Particularly, in order to localize the ground station, global navigation satellite systems (GNSSs) such as the Global Positioning System can be utilized [5]. However, GNSSs operate in the regulated RF band, which requires the installation of additional hardware for the optical ground stations. Moreover, the location error of GNSSs can be up to tens of meters [6], which results in large pointing errors in space optical communications. Alternatively, in the optical band, two techniques are commonly used to obtain the location information. The first method employs a telescope array to form multiple images of celestial objects of known locations. Based on the pixels of the images, the locations of the ground station or spacecraft can be coarsely inferred [4]. Tracking algorithms need to be further applied to improve the location accuracy [7]. The other approach exploits an uplink optical beacon from the ground station: the beacon scans within an uncertainty area and the spacecraft locate the ground station by detecting the beacon using receivers of narrow field-of-view [5], [7]. However, this is only applicable to short-range communications due to the strength limit of the beacon [4]. In addition, neither method estimates the orientation of the ground station or spacecraft, which is crucial to directional space optical communications. Thus, accurate location and orientation estimation techniques that overcome the above shortcomings are much sought after.

In this paper, array processing is employed in space optical communications for the first time to cope with the problems in current localization techniques and improve the estimation accuracy. In subspace-based array processing techniques, the signal from a single source is mapped to a one-dimensional subspace embedded in a high-dimensional complex observation space. Such subspaces of all the sources amount to the 
signal subspace, which is completely determined by the array manifold vectors of the sources. Hence, with the estimate of the signal subspace, the array manifold vectors and their parameters (e.g., the directions of the sources) can be estimated using superresolution techniques like multiple signal classification (MUSIC) and root-MUSIC [8]-[11]. Particularly, in this paper, a novel localization approach is proposed to estimate the location and orientation of the ground station in conjunction with subspace-based array processing techniques. By exploiting the sunlight reflected by a set of celestial objects of known locations, the location and orientation of the ground station can be estimated by solving systems of nonlinear and linear equations. Through computer simulation studies, the proposed approach is shown to effectively estimate the ground station location and orientation with outstanding accuracy and well satisfy the pointing error requirement specified in space optical communications [4].

The organization of the remainder of the paper is as follows. In Section II, the model for the sunlight signal reflected by a set of celestial objects and received by the array of the ground station is presented. In Section III, the proposed localization method is detailed in a four-phase procedure: the directions and ranges of the celestial objects as well as the location and orientation of the ground station are estimated using MUSIC and systems of nonlinear and linear equations. In Section IV, the performance of the proposed algorithm is evaluated via computer simulation studies with respect to the estimation error. Finally, in Section V, the paper is concluded.

\section{Signal Model}

Consider a ground station on Earth that employs an array system of $N$ sensors of a known array geometry. The ground station receives the sunlight reflected by $M$ celestial objects randomly distributed in space of known locations with respect to a global coordinate system. The locations of the ground station and celestial objects are shown in Fig. 1, where the sensors of the ground station are represented by the blue spheres whereas the celestial objects are represented by the red cubes. With reference to Fig. 1, the locations can be specified in two distinct Cartesian coordinate systems: one is the aforementioned global coordinate system $(X, Y, Z)$ while the other is the local coordinate system $(x, y, z)$ with respect to the ground station. Hereafter, for convenience, the terms "global system" and "local system" are used to refer to the global coordinates and local coordinates respectively unless otherwise noted.

In the local system, the known array geometry (namely, the Cartesian coordinates of the sensors) is given as

$$
\left[\underline{r}_{1}, \underline{r}_{2}, \ldots, \underline{r}_{N}\right]=\left[\underline{r}_{x}, \underline{r}_{y}, \underline{r}_{z}\right]^{T} \in \mathcal{R}^{3 \times N}
$$

where $\underline{r}_{k} \in \mathcal{R}^{3 \times 1}$ is the vector with its elements being the Cartesian coordinates of the $k$-th sensor. Without loss of generality, it is assumed that the array centroid is the origin of the local system; i.e., it is the array reference point. Further, the unknown location of the $i$-th celestial object is denoted as $\underline{\underline{r}}_{i} \in \mathcal{R}^{3 \times 1}$. It is parameterized by its unknown

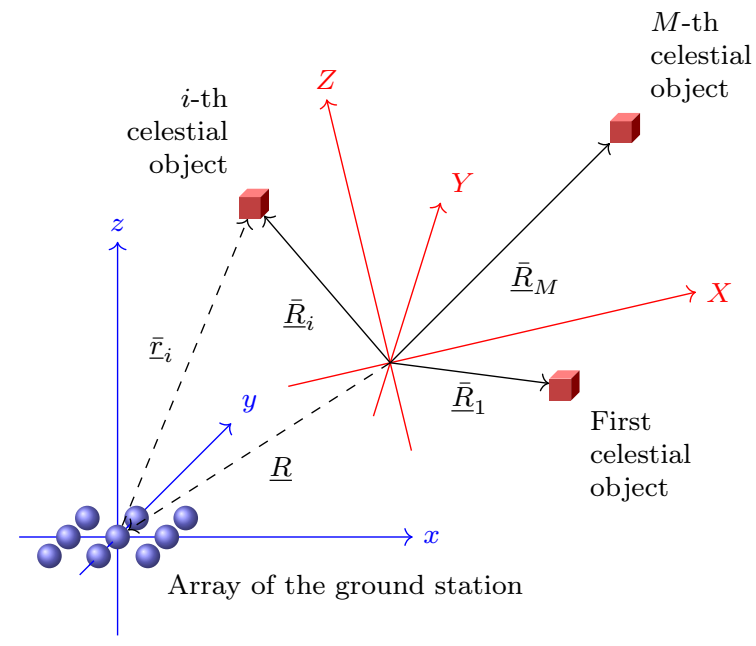

Fig. 1. Geometries of the ground station and celestial objects. The ground station array is represented by the blue spheres and the celestial objects are represented by the red cubes. Their locations can be specified in the local $(x, y, z)$ and global $(X, Y, Z)$ coordinate systems.

azimuth angle $\theta_{i}$ (measured counterclockwise with respect to the positive $x$-axis), elevation angle $\phi_{i}$ (measured with respect to the $x y$-plane), and range $\rho_{i}$ (measured between the array reference point and the celestial object itself) as

$$
\underline{\bar{r}}_{i} \triangleq \underline{\bar{r}}\left(\theta_{i}, \phi_{i}, \rho_{i}\right)=\rho_{i} \underline{u}_{i}
$$

where

$$
\begin{aligned}
\underline{u}_{i} & \triangleq \underline{u}^{\prime}\left(\theta_{i}, \phi_{i}\right) \\
& =\left[\cos \theta_{i} \cos \phi_{i}, \sin \theta_{i} \cos \phi_{i}, \sin \phi_{i}\right]^{T} \in \mathcal{R}^{3 \times 1}
\end{aligned}
$$

is a unit vector pointing from the array reference point towards the celestial object. It is worth noting that $\rho_{i}=\left\|\underline{\underline{x}}_{i}\right\|$.

On the other hand, in the global system, the unknown location of the array system (i.e., the array reference point) is denoted as $\underline{R} \in \mathcal{R}^{3 \times 1}$. In addition, the known location of the $i$-th celestial object in the global system is denoted as $\underline{\underline{R}}_{i} \in \mathcal{R}^{3 \times 1}$.

Furthermore, the unknown mapping from the local system to its global counterpart is governed by a transformation matrix given as

$$
\mathbb{T}=[\mathbb{Q}, \underline{R}] \in \mathcal{R}^{3 \times 4}
$$

where $\mathbb{Q} \in \mathcal{R}^{3 \times 3}$ is the orthogonal rotation matrix and $\underline{R}$, the location of the ground station in the global system, also serves as the translation vector. Using this transformation matrix $\mathbb{T}$, a vector $\underline{v} \in \mathcal{R}^{3 \times 1}$ in the local system can be mapped to its global counterpart $\underline{V} \in \mathcal{R}^{3 \times 1}$ as

$$
\underline{V}=\mathbb{T}\left[\frac{v}{1}\right]=\mathbb{Q} \underline{v}+\underline{R} .
$$

In particular, the rotation matrix $\mathbb{Q}$ can be written in terms of the product of three rotation matrices as

$$
\mathbb{Q}=\mathbb{Q}_{z} \mathbb{Q}_{y} \mathbb{Q}_{x}
$$


where

$$
\begin{aligned}
& \mathbb{Q}_{x}=\left[\begin{array}{ccc}
1, & 0, & 0 \\
0, & \cos \alpha, & \sin \alpha \\
0, & -\sin \alpha, & \cos \alpha
\end{array}\right] \in \mathcal{R}^{3 \times 3} ; \\
& \mathbb{Q}_{y}=\left[\begin{array}{ccc}
\cos \beta, & 0, & -\sin \beta \\
0, & 1, & 0 \\
\sin \beta, & 0, & \cos \beta
\end{array}\right] \in \mathcal{R}^{3 \times 3} ; \\
& \mathbb{Q}_{z}=\left[\begin{array}{ccc}
\cos \gamma, & \sin \gamma, & 0 \\
-\sin \gamma, & \cos \gamma, & 0 \\
0, & 0, & 1
\end{array}\right] \in \mathcal{R}^{3 \times 3}
\end{aligned}
$$

are the respective rotation matrices about the $x-, y$-, and $z$ axis with $\alpha, \beta$, and $\gamma$ being the corresponding unknown Euler angles. By convention, $-\pi \leq \alpha \leq \pi,-\pi / 2 \leq \beta \leq \pi / 2$, and $-\pi \leq \gamma \leq \pi$.

In summary, only the array geometry in the local system and the locations of the celestial objects in the global system are known. The rest are unknown, including the ground station location in the global system $\underline{R}$ and the orientation $\mathbb{Q}$ (or the Euler angles $\alpha, \beta$, and $\gamma$ ), which are to be estimated.

Now, consider the signal model in the local system. The array receives the sunlight reflected by $M$ celestial objects. Note that the solar radiation spans most of the electromagnetic spectrum [12]. Take the strongest visible band as an example; its bandwidth is larger than $300 \mathrm{THz}$, which implies a coherence length shorter than $1 \mu \mathrm{m}$. Thus, it is safe to assume that the sunlight signals traveled along the paths associated with all the celestial objects are incoherent due to the difference in the path distances. In addition, each optical signal can be regarded as a baseband envelope modulated onto an optical carrier; i.e., the component at the center frequency of the optical signal. Therefore, at the ground station, the received baseband signal after downconversion ${ }^{1}$, as illustrated in Fig. 2, can be modeled in a vector format as

$$
\underline{x}(t)=\sum_{i=1}^{M} \underline{S}_{i} m_{i}(t)+\underline{n}(t) \in \mathcal{C}^{N \times 1}
$$

where, for the $i$-th celestial object, $\underline{S}_{i} \in \mathcal{C}^{N \times 1}$ is the plane wave array manifold vector given as

$$
\underline{S}_{i} \triangleq \underline{S}\left(\theta_{i}, \phi_{i}\right)=\exp \left(-j 2 \pi \frac{F_{c}}{c}\left[\underline{r}_{x}, \underline{r}_{y}, \underline{r}_{z}\right] \underline{u}_{i}\right)
$$

with $F_{c}$ being the center frequency of the optical signal and $c$ representing the speed of light. Furthermore, $m_{i}(t)$ is the envelope of the $i$-th celestial object and $\underline{n}(t) \in \mathcal{C}^{N \times 1}$ denotes the complex additive white Gaussian noise of zero mean and unknown power $\sigma_{n}^{2}$. Equation (8) can also be written in a more compact matrix format as

$$
\underline{x}(t)=\mathbb{S} \underline{m}(t)+\underline{n}(t)
$$

\footnotetext{
${ }^{1}$ Lowpass filters can be employed after downconversion to ensure that the envelopes do not vary significantly when traversing across all the elements of the array in addition to remaining incoherent.
}

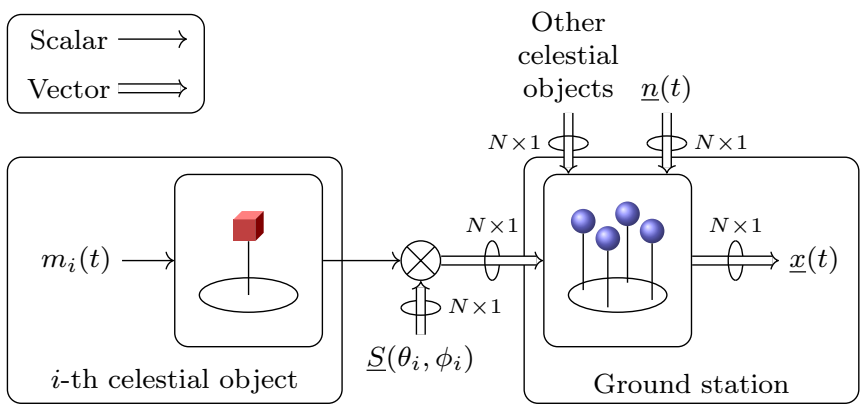

Fig. 2. Baseband representation of the ground station localization system. The signal of the $i$-th celestial object is shown and those of the other celestial objects have a similar structure to the $i$-th one.

where

$$
\begin{aligned}
\mathbb{S} & =\left[\underline{S}_{1}, \underline{S}_{2}, \ldots, \underline{S}_{M}\right] \in \mathcal{C}^{N \times M} ; \\
\underline{m}(t) & =\left[m_{1}(t), m_{2}(t), \ldots, m_{M}(t)\right]^{T} \in \mathcal{C}^{M \times 1} .
\end{aligned}
$$

\section{Proposed Localization Algorithm for the GROUND STATION}

The objective in this paper is to estimate the location of the array reference point of the ground station in the global system as well as the orientation of the local system with respect to the global system. The proposed localization procedure is carried out in the following four phases: First, the azimuth and elevation angles (i.e., the directions of arrival) of all the celestial objects are estimated in the local system using a subspace algorithm like MUSIC. Next, using the estimated directions, the ranges of the celestial objects in the local system are estimated by solving a system of nonlinear equations. Subsequently, in the final two phases, with these range estimates available, the ground station location in the global system as well as its orientation can be estimated by solving a set of linear equations.

\section{A. Phase One: Direction Estimation of Celestial Objects}

Initially, the directions of the celestial objects are estimated based on the second order statistics of the received signal in the local system. In particular, the covariance matrix of the received signal given by Equ. (8) or (10) is

$$
\begin{aligned}
\mathbb{R}_{x x} & =\mathcal{E}\left\{\underline{x}(t) \underline{x}^{H}(t)\right\} \\
& =\mathbb{S R}_{m m} \mathbb{S}^{H}+\mathbb{R}_{n n} \in \mathcal{C}^{N \times N}
\end{aligned}
$$

where $\mathbb{R}_{m m}=\mathcal{E}\left\{\underline{m}(t) \underline{m}^{H}(t)\right\}$ is the covariance matrix of the envelopes and $\mathbb{R}_{n n}=\mathcal{E}\left\{\underline{n}(t) \underline{n}^{H}(t)\right\}=\sigma_{n}^{2} \mathbb{I}_{N}$ is that of the noise. Using MUSIC, the azimuth and elevation angles of the celestial objects can be estimated by evaluating the following cost function

$$
\xi(\theta, \phi)=\frac{\underline{S}^{H}(\theta, \phi) \underline{S}(\theta, \phi)}{\underline{S}^{H}(\theta, \phi) \mathbb{E}_{n} \mathbb{E}_{n}^{H} \underline{S}(\theta, \phi)}
$$

where $\mathbb{E}_{n}$ denotes the eigenspace of $\mathbb{R}_{x x}$ corresponding to its least significant eigenvalues. 


\section{B. Phase Two: Range Estimation of Celestial Objects}

After estimating the azimuth and elevation angles $\theta_{i}$ and $\phi_{i}$ of all the celestial objects, their directional unit vectors $\underline{u}_{i}$ can be derived. Define the matrix containing the estimates of the directional unit vectors of all the celestial objects as

$$
\mathbb{U}=\left[\underline{\hat{u}}_{1}, \underline{\hat{u}}_{2}, \ldots, \underline{\hat{u}}_{M}\right] \in \mathcal{R}^{3 \times M} .
$$

In addition, a vector comprising the unknown ranges of all the celestial objects can be defined as

$$
\underline{\rho}=\left[\rho_{1}, \rho_{2}, \ldots, \rho_{M}\right]^{T} \in \mathcal{R}^{M \times 1} .
$$

Recall that the location of the $i$-th celestial object in the local system is given as $\underline{\underline{r}}_{i}=\rho_{i} \underline{u}_{i}$. This location vector $\underline{\underline{r}}_{i}$ is variant with respect to the change of the coordinate system; i.e., its global counterpart $\underline{R}_{i}$, which is given as

$$
\underline{\bar{R}}_{i}=\mathbb{Q} \underline{\bar{r}}_{i}+\underline{R},
$$

is not equal to $\overline{\underline{x}}_{i}$ in general. However, the difference between any two location vectors $\underline{\underline{x}}_{i}$ and $\overline{\underline{x}}_{j}$ is invariant with respect to the coordinate transformation. Thus, taking all the $J=\left(\begin{array}{c}M \\ 2\end{array}\right)$ pairs of celestial objects into account, this invariance can be represented (see Appendix) as

$$
\left(\mathbb{I}_{J} \otimes \underline{\rho}\right)^{T} \mathbb{A}_{\rho} \underline{\rho}=\underline{b}_{\rho}
$$

where

$$
\begin{aligned}
\mathbb{A}_{\rho}= & {\left[\begin{array}{c}
\mathbb{F}_{12} \mathbb{U}^{T} \mathbb{U F} \mathbb{F}_{12} \\
\mathbb{F}_{13} \mathbb{U}^{T} \mathbb{U F} \mathbb{F}_{13} \\
\vdots \\
\mathbb{F}_{(M-1) M} \mathbb{U}^{T} \mathbb{U F}(M-1) M
\end{array}\right] \in \mathcal{R}^{J M \times M} ; } \\
\underline{b}_{\rho}= & {\left[\begin{array}{c}
\left\|\underline{\underline{R}}_{1}-\underline{\bar{R}}_{2}\right\|^{2} \\
\left\|\underline{\bar{R}}_{1}-\underline{\bar{R}}_{3}\right\|^{2} \\
\vdots \\
\left\|\underline{\bar{R}}_{M-1}-\underline{\bar{R}}_{M}\right\|^{2}
\end{array}\right] \in \mathcal{R}^{J \times 1} }
\end{aligned}
$$

with $\mathbb{F}_{i j} \in \mathcal{R}^{M \times M}$ for $i<j$ being the selection matrix of the $i$-th and $j$-th celestial objects, defined as

$$
\mathbb{F}_{i j}=\operatorname{diag}\left(\left[\underline{0}_{i-1}^{T}, 1, \underline{0}_{j-i-1}^{T},-1, \underline{0}_{M-j}^{T}\right]^{T}\right), \quad i<j .
$$

By solving Equ. (18), which is a set of $J$ quadratic equations of $M$ unknowns where $M>2$ (i.e., $J \geq M$ ), the ranges $\rho$ can be easily estimated ${ }^{2}$.

\section{Phase Three: Location Estimation of Ground Station}

Having estimated the directions and ranges of the celestial objects in the local system, the location of the ground station in the global system should be estimated next. Based on Equ. (17) and [13], the location $\underline{R}$ can be estimated by solving the following set of linear equations

$$
\mathbb{A}_{R} \underline{R}=\underline{b}_{R} \Rightarrow \underline{\hat{R}}=\mathbb{A}_{R}^{\#} \underline{b}_{R}
$$

\footnotetext{
${ }^{2}$ Note that the MATLAB fsolve function can be utilized to solve this system of quadratic equations.
}

where

$$
\begin{aligned}
\mathbb{A}_{R}= & {\left[\begin{array}{c}
2\left(\underline{\bar{R}}_{1}-\underline{\bar{R}}_{2}\right)^{T} \\
2\left(\underline{\bar{R}}_{1}-\underline{\bar{R}}_{3}\right)^{T} \\
\vdots \\
2\left(\bar{R}_{1}-\underline{\bar{R}}_{M}\right)^{T}
\end{array}\right] \in \mathcal{R}^{(M-1) \times 3} } \\
\underline{b}_{R}= & {\left[\begin{array}{c}
\left(\left\|\underline{\bar{R}}_{1}\right\|^{2}-\left\|\underline{\bar{R}}_{2}\right\|^{2}\right)-\left(\hat{\rho}_{1}^{2}-\hat{\rho}_{2}^{2}\right) \\
\left(\left\|\underline{\underline{R}}_{1}\right\|^{2}-\left\|\underline{\bar{R}}_{3}\right\|^{2}\right)-\left(\hat{\rho}_{1}^{2}-\hat{\rho}_{3}^{2}\right) \\
\vdots \\
\left(\left\|\underline{\underline{R}}_{1}\right\|^{2}-\left\|\underline{\underline{R}}_{M}\right\|^{2}\right)-\left(\hat{\rho}_{1}^{2}-\hat{\rho}_{M}^{2}\right)
\end{array}\right] \in \mathcal{R}^{(M-1) \times 1} }
\end{aligned}
$$

with $M>3$ (i.e., $M-1 \geq 3$ ).

\section{Phase Four: Orientation Estimation of Ground Station}

The final step is to estimate the ground station orientation. Equation (17) can be rewritten as

$$
\left(\underline{\bar{r}}_{i} \otimes \mathbb{I}_{3}\right)^{T} \operatorname{vec}(\mathbb{Q})=\underline{\bar{R}}_{i}-\underline{\hat{R}}
$$

where $\operatorname{vec}(\mathbb{Q})$ is the only unknown to be estimated. Likewise, $\operatorname{vec}(\mathbb{Q})$ is also determined by solving the following system of linear equations

$$
\mathbb{A}_{Q} \operatorname{vec}(\mathbb{Q})=\underline{b}_{Q} \Rightarrow \operatorname{vec}(\hat{\mathbb{Q}})=\mathbb{A}_{Q}^{\#} \underline{b}_{Q}
$$

where

$$
\begin{aligned}
\mathbb{A}_{Q} & =\left(\left[\begin{array}{c}
\bar{r}_{1}^{T} \\
\underline{\bar{r}}_{2}^{T} \\
\vdots \\
\overline{\bar{r}}_{M}^{T}
\end{array}\right] \otimes \mathbb{I}_{3}\right) \in \mathcal{R}^{3 M \times 9} ; \\
\underline{b}_{Q} & =\left[\begin{array}{c}
\bar{R}_{1} \\
\overline{\bar{R}}_{2} \\
\vdots \\
\underline{\underline{R}}_{M}
\end{array}\right]-\underline{1}_{M} \otimes \underline{R} \in \mathcal{R}^{3 M \times 1} .
\end{aligned}
$$

with $M>2$ (i.e., $3 M \geq 9$ ).

Further, the Euler angles $\alpha, \beta$, and $\gamma$ can be derived from $\hat{\mathbb{Q}}$ as

$$
\begin{aligned}
& \hat{\alpha}=-\arctan \left(\frac{\underline{F}_{3}^{T} \hat{\mathbb{Q}} \underline{F}_{2}}{\underline{F}_{3}^{T} \hat{\mathbb{Q}} \underline{F}_{3}}\right) ; \\
& \hat{\beta}=\arcsin \left(\underline{F}_{3}^{T} \hat{\mathbb{Q}} \underline{F}_{1}\right) ; \\
& \hat{\gamma}=-\arctan \left(\frac{\underline{F}_{2}^{T} \hat{\mathbb{Q}} \underline{F}_{1}}{\underline{F}_{1}^{T} \hat{\mathbb{Q}} \underline{F}_{1}}\right)
\end{aligned}
$$

where $\underline{F}_{d}=\left[\underline{0}_{d-1}^{T}, 1, \underline{0}_{3-d}^{T}\right]^{T} \in \mathcal{R}^{3 \times 1}$ is the selection vector with $d=1,2,3$. Thus, it is clear that at least four celestial objects are needed to successfully estimate the location and orientation of the ground station.

The proposed localization algorithm can be summarized as the following steps. 
1) Estimate the directions of the celestial objects by using a subspace direction finding algorithm like MUSIC; e.g., Equ. (14).

2) Estimate the ranges of the celestial objects by solving Equ. (18) and selecting the positive solution set.

3) Estimate the ground station location using Equ. (21).

4) Estimate the ground station orientation using Equs. (24) and (26).

Note that in general, an array is utilized to localize sources based on their signals. On the contrary, in this paper, an array is employed to localize itself based on the signals reflected by a set of celestial objects of known locations. The proposed localization algorithm is not limited to space optical communications, and can be applied to any other similar localization problems; e.g., the localization of a spacecraft with the aid of the signals from multiple ground stations of known locations on Earth.

\section{Computer Simulation Studies}

In this section, the performance of the proposed localization algorithm is assessed through computer simulation studies. In the simulations, a $10 \times 10$ grid array of half-wavelength intersensor spacing is utilized at the ground station [14], and four celestial objects are randomly selected in space. The locations of these four celestial objects and the ground station in the local and global systems are listed in Table I. Other array system parameters are listed in Table II. Note that parameters highlighted in gray background are unknown and are to be estimated. Also, the fractional parts of the values in Table I and the Euler angles in Table II are truncated.

Figure 3 shows the computer simulation result of the estimation of the directions of the four celestial objects from the ground station, with the search step size of $1^{\circ}$. Four peaks above $30 \mathrm{~dB}$ can be clearly observed at the azimuth and elevation angles of the celestial objects with some approximation (there are estimation errors due to the $1^{\circ}$ step size). In order to improve the accuracy of the direction estimation, the cost function of Equ. (14) is maximized in the interval of $\pm 1^{\circ}$ of the estimated directions using any convex optimization algorithm ${ }^{3}$. Upon completion of the direction estimation, the ranges of the celestial objects, the location of the ground station in the global system, and its orientation in the format of the Euler angles are estimated subsequently. The estimation errors of all the four phases (including the direction estimation) are listed in Table III. Note that the errors of the direction estimates are small as expected since MUSIC is a superresolution algorithm. Moreover, the errors for the ranges and the location of the ground station are in the order of $1 \times 10^{-2} \mathrm{~m}$ and for the Euler angles $\left(1 \times 10^{-5}\right)^{\circ}$, which suggests that the ground station location and orientation are very accurately estimated. Furthermore, based on the distance between Earth and space (which is longer than $100 \mathrm{~km}$ ), the location estimation error yields a pointing error of less than

\footnotetext{
${ }^{3}$ The MATLAB fmincon function can be utilized in the interval of $\pm 1^{\circ}$ of the estimated directions.
}

TABLE I

Locations of Celestial Objects (CO) And GRound Station (GS)

\begin{tabular}{|c|r|r|r|r|r|r|}
\hline & \multicolumn{3}{|c|}{ Local system } & \multicolumn{3}{c|}{ Global system } \\
\cline { 2 - 7 } & $\theta\left({ }^{\circ}\right)$ & $\phi\left({ }^{\circ}\right)$ & $\rho(\mathrm{m})$ & \multicolumn{1}{c|}{$X(\mathrm{~m})$} & \multicolumn{1}{c|}{$Y(\mathrm{~m})$} & \multicolumn{1}{c|}{$Z(\mathrm{~m})$} \\
\hline $\mathrm{CO} 1$ & 16.15 & 71.95 & 558920 & 437517 & -140664 & 94730 \\
\hline $\mathrm{CO} 2$ & 138.03 & 60.85 & 292239 & 203728 & 169995 & 132296 \\
\hline $\mathrm{CO} 3$ & 277.98 & 57.17 & 103958 & 5386 & 37406 & 215873 \\
\hline $\mathrm{CO} 4$ & 305.96 & 18.05 & 748918 & 31577 & -550666 & 553146 \\
\hline GS & & & & -72397 & 78545 & 160512 \\
\hline
\end{tabular}

TABLE II

ORIENTATION AND OTHER ARRAY SySTEM PARAMETERS

\begin{tabular}{|c|c|c|c|}
\hline Parameter & Value & Parameter & Value \\
\hline Euler angle $\alpha$ & $85.19^{\circ}$ & Carrier frequency $F_{c}$ & $600 \mathrm{THz}$ \\
\hline Euler angle $\beta$ & $-22.12^{\circ}$ & Sampling frequency $F_{s}$ & $3 \mathrm{GHz}$ \\
\hline \multirow[t]{2}{*}{ Euler angle $\gamma$} & $97.28^{\circ}$ & Number of snapshots $L$ & $1 \times 10^{6}$ \\
\hline & & SNR & $30 \mathrm{~dB}$ \\
\hline
\end{tabular}

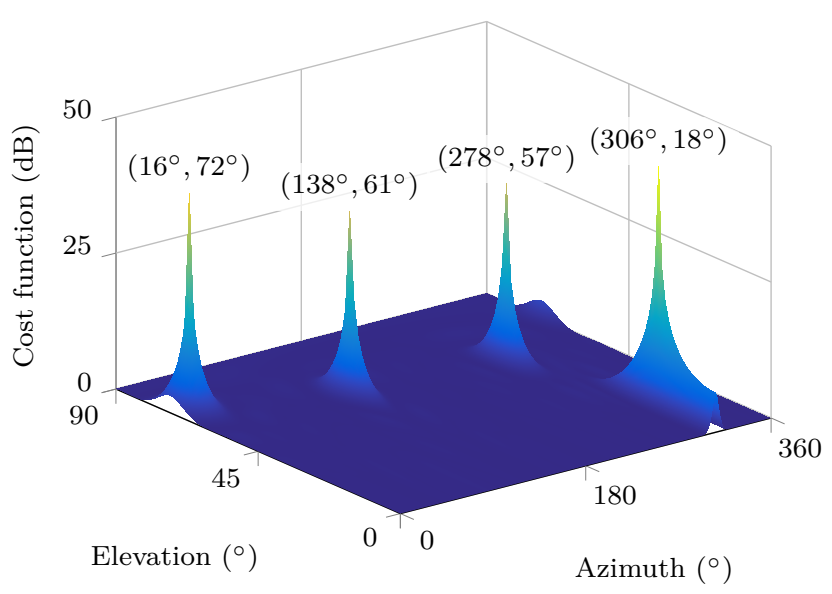

Fig. 3. Direction estimation with the azimuth and elevation angles of the four celestial objects labeled as $(\theta, \phi)$.

1 urad $=\left(5.7296 \times 10^{-5}\right)^{\circ}$, well satisfying the requirement of space optical communications. Note that the estimation precision, which is expected to be high in this kind of applications, can be further improved by increasing the number of snapshots, number of array elements, or array aperture.

The performance of the proposed approach is also evaluated with respect to the root mean squared error (RMSE) of the estimation using Monte Carlo simulation studies. The simulation parameters are the same except that the product of the signalto-noise ratio (SNR) and number of snapshots varies. Figure 4 shows the RMSE curves of the location (displacement) and orientation (Euler angles $\alpha, \beta$, and $\gamma$ ) estimation. The curves decline as $\mathrm{SNR} \times L$ increases. The reason is that with higher SNR or more number of snapshots, the signal subspace can be more accurately reconstructed. This leads to a better estimation of the azimuth and elevation angles of the celestial objects. As the location and orientation estimates of the ground station are 
TABLE III

ESTIMATION ERRORS

\begin{tabular}{|c|c|c|c|}
\hline & $\theta\left(^{\circ}\right)$ & $\phi\left(^{\circ}\right)$ & $\rho(\mathrm{m})$ \\
\hline $\mathrm{CO} 1$ & $0.1336 \times 10^{-5}$ & $3.8740 \times 10^{-6}$ & $3.9252 \times 10^{-2}$ \\
\cline { 2 - 4 } $\mathrm{CO} 2$ & $1.0236 \times 10^{-5}$ & $3.9971 \times 10^{-6}$ & $0.0933 \times 10^{-2}$ \\
\hline $\mathrm{CO} 3$ & $0.8049 \times 10^{-5}$ & $8.4093 \times 10^{-6}$ & $5.2462 \times 10^{-2}$ \\
\hline $\mathrm{CO} 4$ & $0.0390 \times 10^{-5}$ & $8.1366 \times 10^{-6}$ & $3.6386 \times 10^{-2}$ \\
\hline \multirow{2}{*}{$\mathrm{GS}$} & $X(\mathrm{~m})$ & $Y(\mathrm{~m})$ & $Z(\mathrm{~m})$ \\
\cline { 2 - 4 } & $0.6033 \times 10^{-2}$ & $6.9299 \times 10^{-2}$ & $5.5737 \times 10^{-2}$ \\
\hline \multirow{2}{*}{$\begin{array}{c}\text { Euler } \\
\text { angle }\end{array}$} & $\alpha\left(^{\circ}\right)$ & $\beta\left(^{\circ}\right)$ & $\gamma\left({ }^{\circ}\right)$ \\
\cline { 2 - 4 } & $1.2251 \times 10^{-5}$ & $1.9118 \times 10^{-5}$ & $0.0961 \times 10^{-5}$ \\
\hline
\end{tabular}

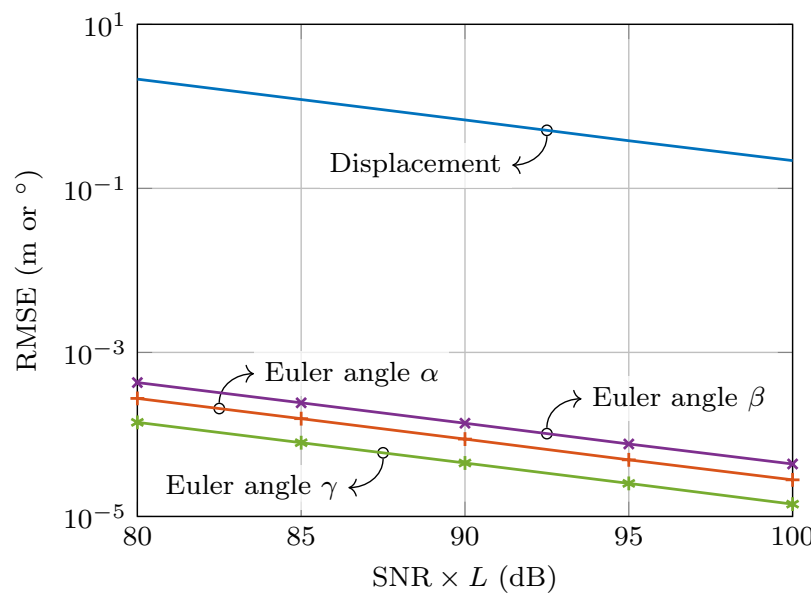

Fig. 4. Location and orientation RMSE over 10000 realizations. The location RMSE is measured in the format of the displacement between the true and estimated locations.

derived from the direction estimates of the celestial objects, their RMSE becomes smaller consequently.

In short, with the exploitation of the proposed approach, the location and orientation of the ground station on Earth can be precisely estimated with exceptional accuracy. Further, the pointing error brought by this algorithm well satisfies the requirement in space optical communications.

\section{CONCLUSIONS}

In this paper, using subspace-based array processing techniques, a novel localization algorithm is proposed for space optical communications to estimate the location and orientation of the ground station in the global coordinate system using the sunlight reflected by at least four celestial objects in space. The performance of the proposed approach is assessed through computer simulation studies. It is shown that the location and orientation of the ground station are successfully estimated via the proposed approach with outstanding accuracy.

\section{APPENDIX}

With reference to Equ. (17), the locations of the $i$-th and $j$-th celestial objects in the global system are given as

$$
\underline{\bar{R}}_{i}=\mathbb{Q} \underline{\underline{r}}_{i}+\underline{R}
$$

$$
\underline{\bar{R}}_{j}=\mathbb{Q}_{j}+\underline{R}
$$

and the difference between them is

$$
\underline{\bar{R}}_{i}-\underline{\bar{R}}_{j}=\mathbb{Q}\left(\underline{\underline{x}}_{i}-\underline{\bar{r}}_{j}\right) .
$$

Squaring the Euclidean norms of both sides of Equ. (28) yields

$$
\left\|\underline{\bar{R}}_{i}-\underline{\bar{R}}_{j}\right\|^{2}=\left(\underline{\bar{r}}_{i}-\underline{\bar{r}}_{j}\right)^{T} \mathbb{Q}^{T} \mathbb{Q}\left(\underline{\bar{r}}_{i}-\underline{\bar{r}}_{j}\right) .
$$

Since $\mathbb{Q}$ is the orthogonal rotation matrix, it is straightforward that $\mathbb{Q}^{T} \mathbb{Q}=\mathbb{I}_{3}$. Hence,

$$
\begin{aligned}
& \left\|\underline{\bar{R}}_{i}-\underline{\bar{R}}_{j}\right\|^{2}=\left(\underline{\bar{r}}_{i}-\underline{\bar{r}}_{j}\right)^{T}\left(\underline{\bar{r}}_{i}-\underline{\bar{r}}_{j}\right) \\
& =\left\|\underline{\bar{r}}_{i}\right\|-2 \underline{\bar{r}}_{i}^{T} \underline{\bar{r}}_{j}+\left\|\underline{\underline{r}}_{j}\right\| \\
& =\rho_{i}^{2}-2 \underline{u}_{i}^{T} \underline{u}_{j} \rho_{i} \rho_{j}+\rho_{j}^{2} \text {. }
\end{aligned}
$$

This is a bivariate quadratic equation and can be rewritten as

$$
\left\|\underline{\bar{R}}_{i}-\underline{\bar{R}}_{j}\right\|^{2}=\underline{\rho}^{T} \mathbb{F}_{i j} \mathbb{U}^{T} \mathbb{U} \mathbb{F}_{i j} \underline{\rho} .
$$

Stacking Equ. (31) for all the pairs of celestial objects gives

$$
\left[\begin{array}{c}
\left\|\underline{\underline{R}}_{1}-\underline{\bar{R}}_{2}\right\|^{2} \\
\left\|\underline{\underline{R}}_{1}-\underline{\bar{R}}_{3}\right\|^{2} \\
\vdots \\
\left\|\underline{\bar{R}}_{M-1}-\underline{\bar{R}}_{M}\right\|^{2}
\end{array}\right]=\left[\begin{array}{c}
\underline{\rho}^{T} \mathbb{F}_{12} \mathbb{U}^{T} \mathbb{U} \mathbb{F}_{12} \rho \\
\underline{\rho}^{T} \mathbb{F}_{13} \mathbb{U}^{T} \mathbb{U} \mathbb{F}_{13} \underline{\rho} \\
\vdots \\
\underline{\rho}^{T} \mathbb{F}_{(M-1) M} \mathbb{U}^{T} \mathbb{U} \mathbb{F}_{(M-1) M}
\end{array}\right]
$$

where the left-hand side is $\underline{b}_{\rho}$ and the right-hand side can be rewritten as $\left(\mathbb{I}_{J} \otimes \underline{\rho}\right)^{T} \mathbb{A}_{\rho} \underline{\rho}$, which result in Equ. (18).

\section{REFERENCES}

[1] V. W. S. Chan, "Optical space communications," IEEE Journal of Selected Topics in Quantum Electronics, vol. 6, no. 6, pp. 959-975, Nov./Dec. 2000.

[2] B. G. Boone et al., "Optical communications development for spacecraft applications," Johns Hopkins APL Technical Digest, vol. 25, no. 4, pp. 306-315, Oct.-Dec. 2004.

[3] M. Toyoshima, "Trends in satellite communications and the role of optical free-space communications," Journal of Optical Networking, vol. 4, no. 6, pp. 300-311, Jun. 2005.

[4] H. Hemmati, Ed., Deep Space Optical Communications. Hoboken, NJ: John Wiley \& Sons, 2006.

[5] F. Fidler et al., "Optical communications for high-altitude platforms," IEEE Journal of Selected Topics in Quantum Electronics, vol. 16, no. 5, pp. 1058-1070, Sep./Oct. 2010.

[6] Global Positioning System Standard Positioning Service Performance Standard, Sep. 2008.

[7] B. L. Ulich, "Overview of acquisition, tracking, and pointing system technologies," in Proceedings of SPIE, Acquisition, Tracking, and Pointing II, Los Angeles, CA, 1988, pp. 40-63.

[8] R. O. Schmidt, "Multiple emitter location and signal parameter estimation," IEEE Transactions on Antennas and Propagation, vol. 34, no. 3, pp. 276-280, Mar. 1986.

[9] H. Krim and M. Viberg, "Two decades of array signal processing research: The parametric approach,' IEEE Signal Processing Magazine, vol. 13, no. 4, pp. 67-94, Jul. 1996.

[10] A. Manikas, Differential Geometry in Array Processing. London, UK: Imperial College Press, 2004.

[11] J. Zhuang, W. Li, and A. Manikas, "Fast root-MUSIC for arbitrary arrays," Electronics Letters, vol. 46, no. 2, pp. 174-176, Jan. 2010.

[12] J. A. Duffie and W. A. Beckman, Solar Engineering of Thermal Processes, 4th ed. Hoboken, NJ: Wiley, 2013.

[13] A. Manikas, Y. I. Kamil, and M. Willerton, "Source localization using sparse large aperture arrays," IEEE Transactions on Signal Processing, vol. 60, no. 12, pp. 6617-6629, Dec. 2012.

[14] O. Hayden, R. Agarwal, and C. M. Lieber, "Nanoscale avalanche photodiodes for highly sensitive and spatially resolved photon detection," Nature Materials, vol. 5, pp. 352-356, May 2006. 